\title{
Endocytosis in the mouse oocyte and its contribution to cAMP signaling during meiotic arrest
}

\author{
Katie M Lowther, Viacheslav O Nikolaev ${ }^{1}$ and Lisa M Mehlmann \\ Department of Cell Biology, University of Connecticut Health Center, Farmington, Connecticut 06030, USA and \\ ${ }^{1}$ Heart Research Center Goettingen, Georg August University Medical Center, D-37075 Goettingen, Germany \\ Correspondence should be addressed to L M Mehlmann; Email: Imehlman@neuron.uchc.edu
}

\begin{abstract}
Mammalian oocytes are arrested at prophase I of meiosis until a preovulatory surge of LH stimulates them to resume meiosis. Prior to the LH surge, high levels of cAMP within the oocyte maintain meiotic arrest; this cAMP is generated in the oocyte through the activity of the constitutively active, $\mathrm{G}_{\mathrm{s}}$-coupled receptor, G-protein-coupled receptor 3 (GPR3) or GPR12. Activated GPRs are typically targeted for desensitization through receptor-mediated endocytosis, but a continuously high level of cAMP is needed for meiotic arrest. The aim of this study was to examine whether receptor-mediated endocytosis occurs in the mouse oocyte and whether this could affect the maintenance of meiotic arrest. We found that constitutive endocytosis occurs in the mouse oocyte. Inhibitors of receptor-mediated endocytosis, monodansylcadaverine and dynasore, inhibited the formation of early endosomes and completely inhibited spontaneous meiotic resumption. A red fluorescent protein-tagged GPR3 localized in the plasma membrane and within early endosomes in the oocyte, demonstrating that GPR3 is endocytosed. However, overexpression of G-protein receptor kinase 2 and $\beta$-arrestin-2 had only a modest effect on stimulating meiotic resumption, suggesting that these proteins do not play a major role in GPR3 endocytosis. Inhibition of endocytosis elevated cAMP levels within oocytes, suggesting that there is an accumulation of GPR3 at the plasma membrane. These results show that endocytosis occurs in the oocyte, leading to a decrease in cAMP production, and suggest that there is a balance between cAMP production and degradation in the arrested oocyte that maintains cAMP levels at an appropriate level during the maintenance of meiotic arrest.

Reproduction (2011) 141 737-747
\end{abstract}

\section{Introduction}

Mammalian oocytes become arrested at prophase I of meiosis during embryonic development. Oocytes remain in prophase I until a preovulatory surge of $\mathrm{LH}$ from the pituitary signals to the oocyte, via the surrounding follicle cells, to resume meiosis and progress to metaphase II, where they undergo a second arrest. It is at the MIl stage that fertilization occurs. Meiotic resumption is coordinated with the LH surge, such that by the time ovulation occurs the oocyte has undergone nuclear maturation as well as changes in intracellular organization (cytoplasmic maturation) that prepare the mature, MII-stage oocyte to be fertilized and initiate embryonic development.

It is well established that maintenance of meiotic arrest prior to the LH surge depends on high levels of cAMP in the oocyte (Conti et al. 2002, Mehlmann 2005a). In rodents, cAMP is produced in the oocyte by the activity of a constitutively active G-protein-coupled receptor 3 (GPR3; mouse) or GPR12 (rat; Mehlmann et al. 2004, Hinckley et al. 2005, Ledent et al. 2005, Vaccari et al. 2008), both of which stimulate a $G_{\alpha s}$
G-protein to activate adenylate cyclase and thereby produce cAMP (Horner et al. 2003, Freudzon et al. 2005, Hinckley et al. 2005, Mehlmann 2005b). The removal or inactivation of any of the components of this signaling pathway causes the oocyte to undergo meiotic resumption in the absence of the LH surge (Mehlmann et al. 2002, 2004, 2005b, Horner et al. 2003, Ledent et al. 2005, DiLuigi et al. 2008, Vaccari et al. 2008).

Most GPRs are inactive until they are stimulated by a ligand. Ligand binding triggers a conformational change in the receptor and allows the phosphorylation by G-protein receptor kinases (GRKs) and subsequently the recruitment of $\beta$-arrestins, which cause receptor internalization through clathrin-coated pits. The receptor can then be degraded or recycled back to the membrane (Reiter \& Lefkowitz 2006, Moore et al. 2007). GPR3 is unusual, as it is constitutively active and is likely to signal in the absence of a ligand (Freudzon et al. 2005, Yin et al. 2009). As a constitutively active receptor, it is unclear whether GPR3 is internalized in a similar fashion, and if so, the receptor continues to signal internally to provide a constant level of cAMP to the interior of the oocyte. Recently, the TSH and PTH 
receptors, both of which act through the $\mathrm{G}_{\mathrm{s}}$ signaling pathway, have been shown to continue to signal following endocytosis, within early endosomes (Calebiro et al. 2009, 2010, Ferrandon et al. 2009). In addition, GPR6, which is a constitutively active receptor closely related to GPR3 (Kostenis 2004), is localized within cells rather than on the cell surface, suggesting that it signals within endosomes (Padmanabhan et al. 2009). Signaling within endosomes has been associated with the ability of the cell to maintain a prolonged response to hormone following receptor internalization. Because prolonged cAMP signaling is crucial for the maintenance of meiotic arrest, it is possible that endosomal signaling is required for sustained CAMP production. Alternatively, it is possible that GPR3 must continuously remain in the oocyte plasma membrane in order to be active, so that it would either not recycle or be endocytosed and rapidly recycled back to the membrane. Depletion of GPR3 in the follicle-enclosed mouse oocyte by RNAi causes oocytes to undergo spontaneous maturation in the absence of LH (Mehlmann 2005b), indicating that GPR3 endocytosis and degradation occur in the oocyte, concomitant with synthesis of new GPR3 and insertion into the plasma membrane.

Receptor-mediated endocytosis is a critical process utilized by oocytes of non-mammalian species, such as insects and frogs, to import yolk protein precursors (Opresko \& Wiley 1987). Import of vitellogenin occurs during oocyte growth through clathrin-mediated endocytosis following binding to a receptor. Although mammalian oocytes do not produce yolk, it is possible that endocytosis could be important during oocyte growth. In addition, endocytosis of cell surface receptors could be important for the regulation of meiosis. Indeed, receptor trafficking is critical for the regulation of meiotic maturation in oocytes of Caenorhabditis elegans (Cheng et al. 2008).

Previous studies have shown that vesicular trafficking at the plasma membrane has an important role in maintaining meiotic arrest in Xenopus oocytes (El-Jouni et al. 2007). Inhibiting exocytosis stimulated meiotic resumption in the absence of progesterone, whereas inhibiting endocytosis interfered with the ability of progesterone to stimulate meiotic resumption, indicating that some component that maintains meiotic arrest must be present in the plasma membrane. It was hypothesized that this component could be a constitutively active receptor, although the role of the receptor was not investigated. In this study, we examined whether active endocytosis occurs in the mouse oocyte and whether endocytosis impacts the maintenance of meiotic arrest. Our results demonstrate that trafficking at the membrane occurs and suggest that GPR3 signaling at the plasma membrane, rather than intracellularly, is necessary for the maintenance of meiotic arrest.

\section{Results}

\section{Components of endocytosis are expressed in mouse oocytes}

Endocytosis of receptors in somatic cells occurs predominantly through the clustering and internalization of an activated receptor into clathrin-coated pits (Moore et al. 2007, Wolfe \& Trejo 2007). However, little is known about the identity and function of recycling components in mammalian oocytes. Therefore, we first examined whether two of the most common proteins responsible for receptor endocytosis in somatic cells were expressed in mouse oocytes. Clathrin is a protein that plays an important role in the formation of coated pits in which receptors accumulate (Doherty \& McMahon 2009). The heavy chain of clathrin is present in mouse oocytes, as determined by western blotting. Indeed, we were able to detect clathrin in as few as ten oocytes (Fig. 1A), indicating that it is abundant. Receptor endocytosis also requires the activity of dynamin, a GTPase that is necessary for excising clathrin-coated pits from the plasma membrane (Moore et al. 2007). We detected the expression of the ubiquitously expressed dynamin 2 in the oocyte. In contrast, neither dynamin 1, which is restricted to the nervous system, nor the more

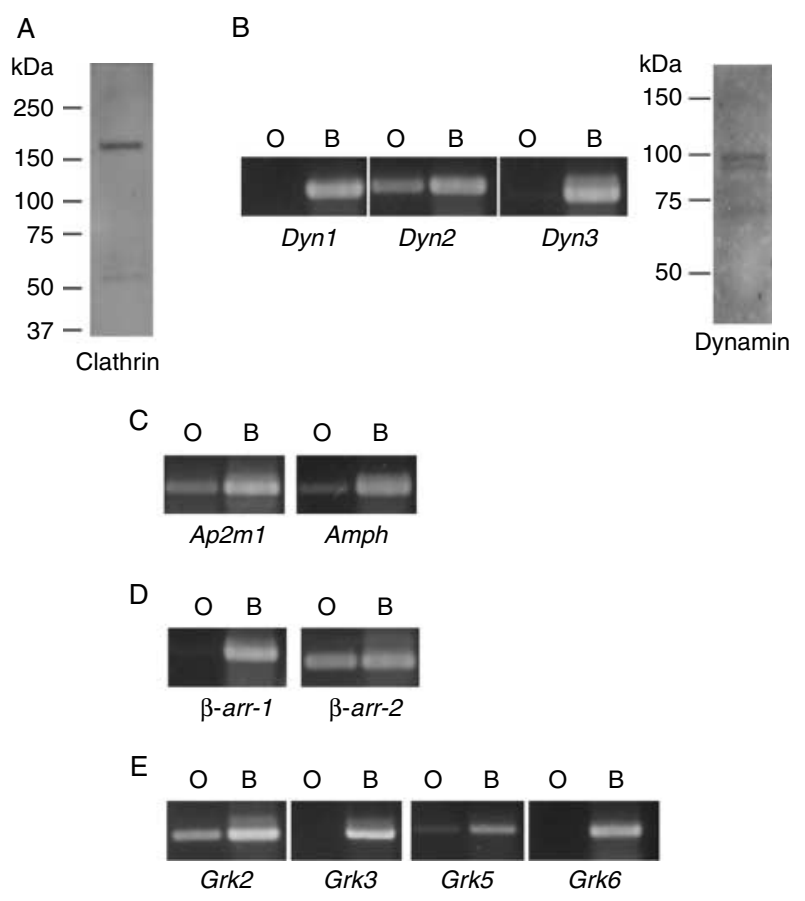

Figure 1 Components of receptor-mediated endocytosis are expressed in mouse oocytes. (A) Expression of clathrin protein, as assessed by western blot using lysate from ten oocytes. (B) Expression of dynamin in mouse oocytes, as assessed by RT-PCR. O, oocyte; B, brain, used as a positive control. (C) Western blot showing two bands at $\sim 100 \mathrm{kDa}$ in lysate from 300 oocytes, demonstrating that dynamin protein is present in mouse oocytes. (C, D and E) RT-PCRs showing expression of other components of endocytosis in mouse oocytes. Ap2m1, AP-2 $\mu 2$ subunit; Amph, amphiphysin; $\beta$-arr, $\beta$-arrestin. 
ubiquitously expressed dynamin 3, was detectable by RT-PCR using our cycling parameters (Fig. 1B). Western blot analysis of oocyte lysate detected two bands at the expected size of $\sim 100 \mathrm{kDa}$, showing that the protein is expressed. The signal was not strong, even using lysate from $500+$ oocytes, suggesting that the amount of dynamin in the oocyte is low. It is unclear why the protein was expressed as a doublet; it is possible that the two bands could represent different splice variants of dynamin 2 (Liu et al. 2008).

In addition to clathrin and dynamin, other major components of receptor recycling include the adaptor proteins AP-2 and amphiphysin, as well as the mediators of endocytosis, $\beta$-arrestins and GRKs. We examined the expression of mRNAs encoding these proteins using RT-PCR. mRNA for one of the major AP-2 subunits, $\mu 2$ (Ap2m1) subunit of AP-2, and amphiphysin were expressed (Fig. 1C). In addition, $\beta$-arrestin-2 and GRK2 and GRK5 were present (Fig. $1 \mathrm{D}$ and $\mathrm{E}$ ), whereas $\beta$-arrestin-1 and Grk3 and Grk6 RNAs were not detectable in oocytes. The visual GRK1 and GRK7, and the testis-specific GRK4, were not examined.

\section{Monodansylcadaverine and dynasore inhibit endocytosis in mouse oocytes}

As a prelude to examining whether endocytosis could have a role in meiotic regulation, we first determined that inhibitors of receptor-mediated endocytosis could prevent endocytosis in mouse oocytes using the inhibitors monodansylcadaverine (MDC) and dynasore (Macia et al. 2006). We used pharmacological inhibitors because in initial experiments we found that we were unable to deplete the amount of clathrin protein using RNAi, nor were we able to significantly deplete the amount of dynamin 2 RNA within our culture period of 4 days using follicle-enclosed oocytes (not shown). We chose compounds that inhibit endocytosis through two different mechanisms: MDC inhibits the protein cross-linker, tissue transglutaminase (Davies et al. 1980), which is thought to inhibit the clustering and internalization of clathrin (Schlegel et al. 1982), whereas dynasore inhibits the GTPase activity of dynamin without affecting the activity of other small GTPases (Macia et al. 2006).

To confirm that MDC blocked endocytosis in oocytes, we treated oocytes with FM 1-43, a dye that becomes fluorescent when it incorporates into membranes and retains its fluorescence when membrane is endocytosed. Following a $3 \mathrm{~h}$ culture period in the presence of $100 \mu \mathrm{M}$ MDC and $10 \mu \mathrm{M}$ milrinone (to prevent spontaneous oocyte maturation), we incubated zona-free oocytes in FM 1-43 and examined them with a confocal microscope 1-2 h later. Zona-free oocytes were used because the FM 1-43 labeled transzonal processes in the zona pellucida, making plasma membrane fluorescence difficult to quantify. Untreated oocytes were fluorescent in the plasma membrane and throughout the cytoplasm, often in punctate clusters (Fig. 2A). In contrast, oocytes preincubated in MDC were mainly fluorescent in the plasma membrane. The plasma membrane labeling was more intense than that in controls, suggesting that the treated oocytes had more membrane area due to the absence of endocytosis (Fig. 2A). The total plasma membrane to cytoplasmic fluorescence ratio was significantly higher in oocytes treated with MDC than in controls $(P<0.0001$; Fig. 2B), demonstrating an inhibition of endocytosis. We also attempted to examine whether dynasore inhibits endocytosis in oocytes using FM 1-43. However, plasma membrane labeling in the presence of dynasore was very faint, indicating that dynasore might interfere with the incorporation of FM 1-43 into the membrane.

Endocytosed proteins enter early endosomes, after which they can be sorted into late endosomes and lysosomes, or can be recycled back to the membrane. We hypothesized that inhibiting endocytosis with MDC and dynasore would block the formation of early endosomes. For these experiments, we labeled early endosomes by immunofluorescence using a specific antibody against the early endosome marker, early endosome antigen 1 (EEA1; Fig. 2C). In control oocytes, early endosomes were abundantly present in both the cortex and throughout the cytoplasm, excluding the germinal vesicle (GV; Fig. 2C). The diameter of the fluorescent spots was generally much larger than the $50-400 \mathrm{~nm}$ diameter that characterizes endosomes in most somatic cells (Jovic et al. 2010), reaching sizes of up to $\sim 5 \mu \mathrm{m}$ (Fig. 2C). MDC dose dependently decreased the number of endosomes present in the oocyte following a $3 \mathrm{~h}$ incubation, such that at a concentration of $100 \mu \mathrm{M}$, early endosomes were completely absent (Fig. 2C). A similar reduction in early endosomes was seen using $80 \mu \mathrm{M}$ dynasore, a concentration commonly used to inhibit endocytosis in somatic cells (Macia et al. 2006, Newton et al. 2006, de Beco et al. 2009, Barrias et al. 2010; Fig. 2C). These results show that there is rapid endocytosis at the plasma membrane in mouse oocytes and that both MDC and dynasore effectively inhibit endocytosis.

\section{Inhibiting endocytosis prevents spontaneous meiotic resumption in mouse oocytes}

To examine the effect of inhibiting endocytosis on spontaneous meiotic resumption, oocytes were pretreated with MDC or dynasore for $3 \mathrm{~h}$ in the presence of milrinone, then were washed out of milrinone while maintained in MDC or dynasore, and were periodically scored for the presence of a GV. MDC dose dependently inhibited spontaneous meiotic resumption for at least $7 \mathrm{~h}$ after oocytes were washed out of milrinone (Fig. 3A). Oocytes became unhealthy during overnight incubation in MDC, so later time points were not examined. Similar to MDC, $80 \mu \mathrm{M}$ dynasore dose 


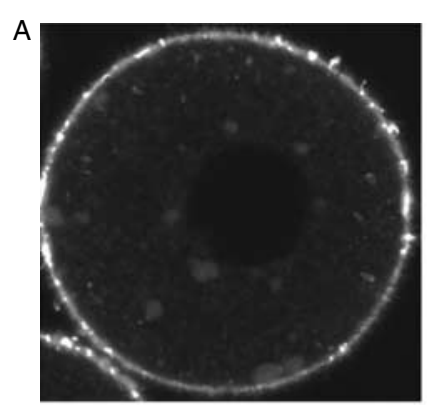

Control

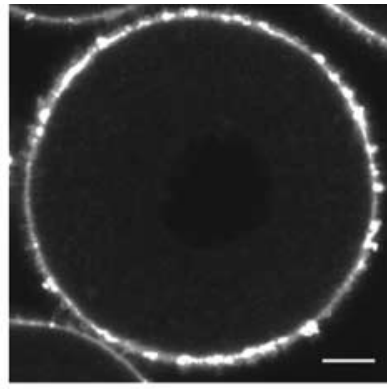

MDC
B

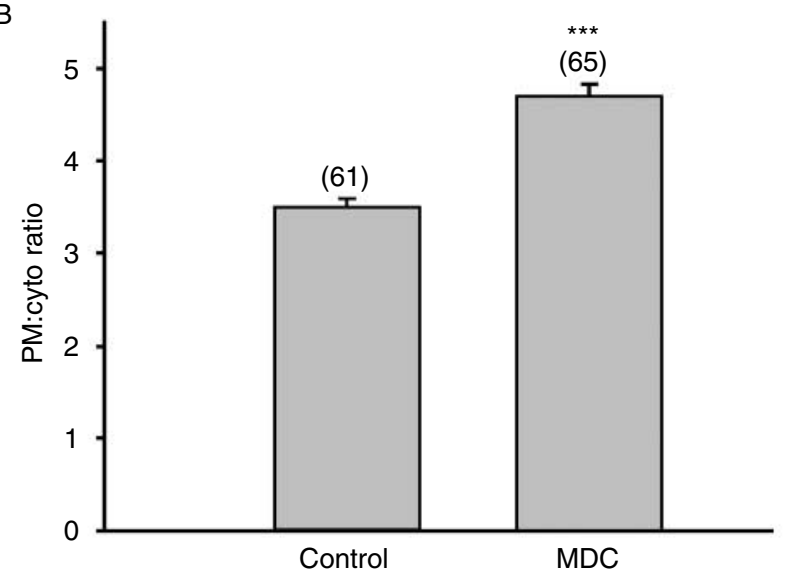

C

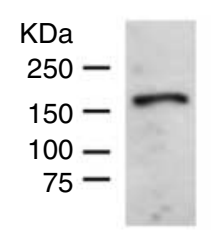

EEA1

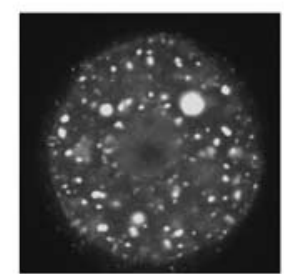

Untreated

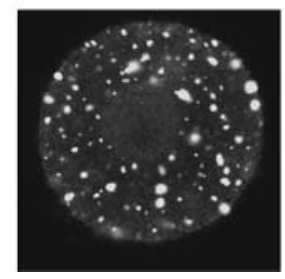

Untreated

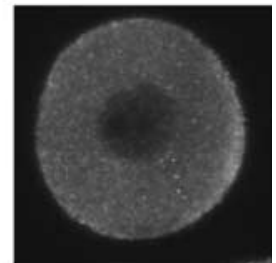

MDC

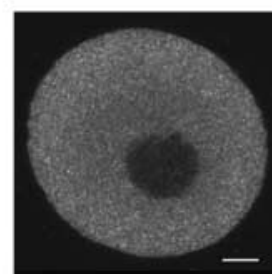

Dynasore
Figure 2 Inhibitors of clathrin-mediated endocytosis inhibit endocytosis in mouse oocytes. (A) MDC inhibits endocytosis. Isolated oocytes were treated with $100 \mu \mathrm{M} \mathrm{MDC}$, the zonae were removed, and oocytes were incubated in $2 \mu \mathrm{M}$ FM 1-43 and imaged 1-2 h later with a confocal microscope. (B) Quantification of membrane to cytoplasmic fluorescence in control and MDC-treated oocytes. The membrane to cytoplasm ratio was significantly higher in MDC-treated than in control oocytes $\left({ }^{* * *} P<0.0001\right.$; error bars $=$ mean \pm s.E.M.). The number of oocytes is indicated in parentheses. (C) MDC and dynasore block the formation of early endosomes. (Left) Antibody against EEA1 that was used for these experiments specifically recognizes a single band from mouse oocyte lysate. (Right) Early endosomes are present throughout the oocyte excluding the GV in control oocytes, whereas in oocytes treated with $100 \mu \mathrm{M}$ MDC or $80 \mu \mathrm{M}$ dynasore early endosomes were completely absent. Bars $=10 \mu \mathrm{m}$. dependently inhibited spontaneous maturation in the absence of milrinone (Fig. 3B).

The effects of MDC and dynasore were reversible, as oocytes treated with MDC and dynasore for $3 \mathrm{~h}$ underwent spontaneous maturation following the removal of MDC, dynasore, and milrinone (Fig. 3C). There was a slight delay between the time the control oocytes underwent GVBD and those treated with MDC or dynasore. Although almost half of the control oocytes underwent GVBD within $1 \mathrm{~h}$ following milrinone removal, GVBD was only observed after $2 \mathrm{~h}$ in oocytes treated with MDC or dynasore. The overall rate of GVBD was slower in the group treated with dynasore than in the group treated with MDC. The reason for this delay is unclear. However, almost $100 \%$ of the oocytes in both treated groups underwent GVBD within $5 \mathrm{~h}$ after inhibitor washout, and $84 \%$ of the matured oocytes from the MDC group and $90 \%$ of matured oocytes from the dynasore group formed first polar bodies $(n=25$ and 30 oocytes respectively).

To further characterize MDC and dynasore as reversible endocytosis inhibitors, we examined whether early endosomes reappear following MDC or dynasore washout. Oocytes were incubated in $100 \mu \mathrm{M}$ MDC or $80 \mu \mathrm{M}$ dynasore for $3 \mathrm{~h}$ in the presence of milrinone and then were washed into medium without MDC or dynasore. We kept milrinone in the culture medium for this experiment to rule out an effect of falling cAMP levels (caused by activation of phosphodiesterase (PDE; Norris et al. 2009)) on the localization of endosomes. Early endosomes, which were completely absent in oocytes treated with MDC or dynasore (Figs. 2D and $3 \mathrm{D})$, began to reappear within $1 \mathrm{~h}$ following MDC or dynasore washout (Fig. 3D). Endosomes were generally smaller than those seen in controls and were often concentrated around the cortex, where early endosomes are formed. The formation of early endosomes preceded GVBD in oocytes treated with MDC or dynasore, indicating that CAMP levels are higher in the oocyte when endocytosis is blocked and then fall when endocytosis resumes.

To examine the possibility that falling cAMP levels due to removal of milrinone from medium that did not contain endocytosis inhibitors affects endosome localization, we collected oocytes in the presence of milrinone and labeled early endosomes 1 and $2 \mathrm{~h}$ following milrinone washout. Localization of endosomes was indistinguishable from those observed in the presence of milrinone (not shown), demonstrating that endosome formation is independent of cAMP levels in the oocyte.

\section{GPR3 undergoes endocytosis in the oocyte}

Meiotic arrest depends on high levels of cAMP in the oocyte (Mehlmann 2005a). Because GPR3 is responsible for maintaining meiotic arrest prior to the $\mathrm{LH}$ surge, we 
A

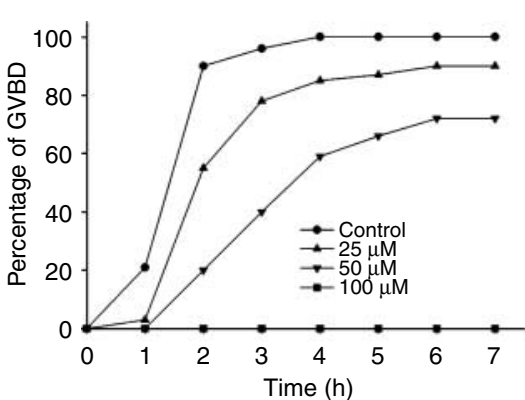

C

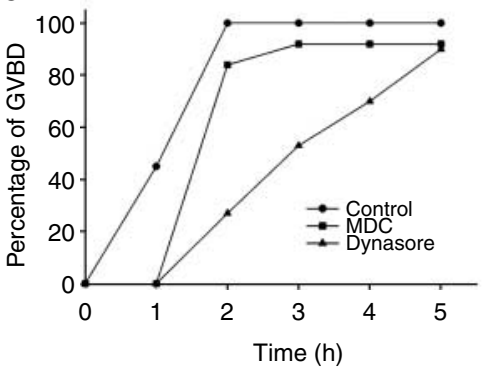

B

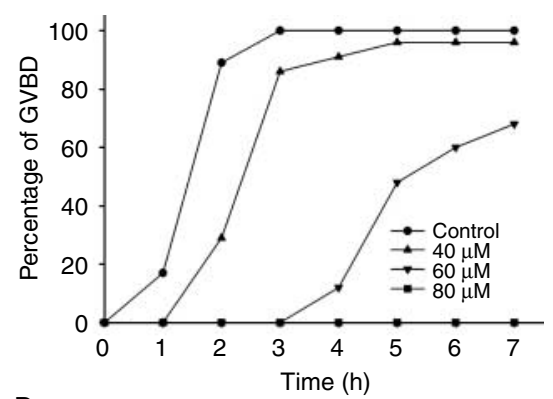

D

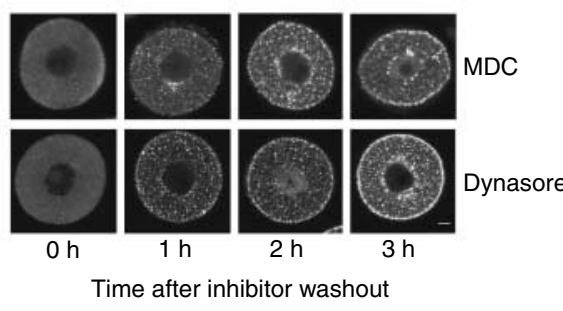

Figure $3 \mathrm{MDC}$ and dynasore inhibit spontaneous meiotic resumption in oocytes. Isolated oocytes were treated with the indicated concentrations of MDC (A) or dynasore (B). After a $3 \mathrm{~h}$ incubation period, the oocytes were washed out of milrinone and periodically scored for the presence or absence of a GV. (C) The effects on of MDC and dynasore on inhibiting GVBD are reversible. Oocytes were treated with MDC or dynasore for $3 \mathrm{~h}$ in the presence of milrinone and were washed out of all inhibitors. (D) Early endosomes, which were absent following a $3 \mathrm{~h}$ treatment with MDC or dynasore, re-form within $1 \mathrm{~h}$ after MDC or dynasore are removed from the culture medium. $\mathrm{Bar}=10 \mu \mathrm{m}$. were interested in knowing whether its localization is important for the regulation of meiotic arrest. Most GPRs are internalized following agonist stimulation. However, as a constitutively active receptor, it is possible that GPR3 does not get internalized but remains at the plasma membrane and continuously signals. We examined GPR3 internalization in the oocyte by expressing GPR3 fused to red fluorescent protein (GPR3-RFP). Overexpressed GPR3-RFP localized both in the plasma membrane and in clusters throughout the cytoplasm of the oocyte (Fig. 4) when incubated in the presence of milrinone. Double labeling with EEA1 showed that GPR3-RFP co-localized within early endosomes. These results demonstrate that GPR3 is internalized in the mouse oocyte.

We attempted to examine the localization of GPR3RFP in response to treatment of MDC and dynasore. However, there was no noticeable accumulation of GPR3-RFP in the plasma membrane in response to inhibitor treatment (not shown). This could be due to the large surface area of the oocyte and the high level of membrane labeling of overexpressed GPR3-RFP that would not permit the detection of small changes in plasma membrane fluorescence.

\section{Overexpression of $\beta$-arrestin-2 and GRK2 into follicle-enclosed oocytes has a partial effect on stimulating GVBD}

If GPR3 signaling at the plasma membrane is necessary to maintain meiotic arrest, then the removal of GPR3 from the membrane would be expected to cause GVBD. Receptor-mediated endocytosis generally occurs by phosphorylation of an activated receptor by GRKs, followed by binding to $\beta$-arrestins, which remove the receptor from the membrane and sort it into clathrincoated pits (Reiter \& Lefkowitz 2006, Moore et al. 2007). We examined whether GRKs/ $\beta$-arrestins are involved in GPR3 endocytosis by determining the effect on GVBD of overexpressing $\beta$-arrestin-2 and GRK2 in follicleenclosed oocytes. Overexpression of $\beta$-arrestins/GRKs hyperphosphorylates and enhances endocytosis of a constitutively active viral receptor, US28, in somatic cells and decreases its activity (Miller et al. 2003). We used follicle-enclosed oocytes for these experiments because unlike the above pharmacological inhibitors, which are cell permeable, the GRK2 and $\beta$-arrestin-2GFP proteins were not permeable, thereby allowing us to discern an effect of the proteins on the oocyte as opposed to the follicle. In addition, use of follicle-enclosed oocytes simulates the native environment of the oocyte, rather than using oocytes incubated in inhibitors that artificially raise cAMP levels.

We microinjected RNA encoding a fluorescently tagged $\beta$-arrestin-2 ( $\beta$-arrestin-2-GFP) and GRK2 into oocytes from follicles $\geq 320 \mu \mathrm{m}$ in diameter; such follicles contain oocytes that are competent to resume meiosis. After a 17-24 h incubation to allow time for the proteins to be expressed, oocytes were removed from their follicles and the meiotic status evaluated. By the end of the culture period, $13 \%$ of the injected oocytes had undergone GV breakdown (GVBD) compared with $0 \%$ of control oocytes ( $n=24$ and $n=23$ respectively; Fig. 5A), despite the proteins being robustly expressed as determined by western blot for GRK2 (Fig. 5B) and fluorescence microscopy for $\beta$-arrestin-2-GFP (not shown).

To confirm that our $\beta$-arrestin-2 and/or GRK2 proteins were active, we examined the ability of $\beta$-arrestin-2-GFP to translocate from the cytoplasm to the plasma membrane following stimulation of an exogenously 


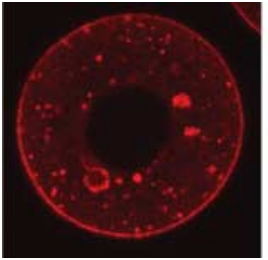

GPR3-RFP

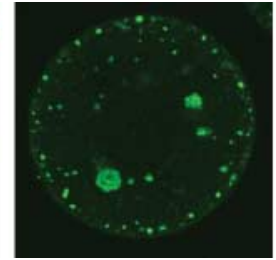

EEA1

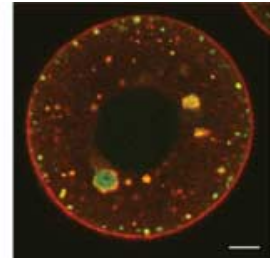

Merge
Figure 4 GPR3-RFP localizes in the plasma membrane in oocytes and co-localizes with early endosomes. Isolated oocytes were injected with RNA encoding GPR3-RFP and cultured overnight prior to fixation. Early endosomes were labeled using immunofluorescence. GPR3-RFP (left). Early endosomes (middle). Merge (right). Bar $=10 \mu \mathrm{m}$.

expressed $\mathrm{G}_{\mathrm{s}}$-coupled receptor. To do this, we co-injected isolated oocytes with RNA encoding the $\beta 2$ adrenergic receptor $(\beta 2 \mathrm{AR}), \mathrm{GRK} 2$, and $\beta$-arrestin-2GFP. Following an overnight culture to allow time for the oocytes to synthesize the proteins, we examined the oocytes before and after stimulating the receptor with $10 \mu \mathrm{M}$ isoproterenol. Before adding the isoproterenol, oocytes were fluorescent throughout the cytoplasm, excluding the GV (Fig. 5B). Within $2 \mathrm{~min}$ of adding isoproterenol, the $\beta$-arrestin-2-GFP translocated to the plasma membrane (Fig. 5C). The localization to the membrane was transient, and fluorescence began to be lost from the membrane and become punctate within 10 min after adding agonist (Fig. 5C), indicating that the receptor was rapidly removed from the membrane. These results show that mouse oocytes are capable of undergoing GRK/ $\beta$-arrestin-dependent receptor recycling but suggest that if GRKs/ $\beta$-arrestins have a role in clathrin-mediated receptor endocytosis of GPR3, it is not likely to be the primary mechanism by which this occurs in the oocyte.

\section{Inhibiting endocytosis raises cAMP levels in the oocyte}

If GPR3 signaling at the cell membrane is necessary for maintaining meiotic arrest, we would expect to see an increase in CAMP concentration in oocytes in which endocytosis is inhibited. To examine the possibility that GPR3 activity at the plasma membrane increases CAMP levels, we measured cAMP levels in oocytes treated with dynasore. We used dynasore for this experiment because we found in preliminary experiments that MDC interfered with the cAMP sensor, whereas dynasore did not (not shown). To measure cAMP levels, we used a fluorescence resonance energy transfer (FRET)-based assay that has previously been described (Nikolaev et al. 2004, Norris et al. 2009). This assay uses an indicator consisting of yellow fluorescent protein (YFP) and cyan fluorescent protein (CFP) fused to the cAMP-binding domain of EPAC2 (Epac2-camps300). cAMP levels are inversely proportional to FRET when CFP is excited with a laser (see Materials and Methods).
We microinjected the cAMP indicator protein, Epac2camps300, into oocytes and measured the YFP/CFP ratio following excitation of CFP, after treatment with dynasore. We found that oocytes held in the presence of milrinone throughout the course of the experiment maintained a constant fluorescence ratio (Fig. 6). When oocytes were washed out of milrinone, the YFP/CFP ratio increased by $\sim 20 \%$, demonstrating that cAMP levels decreased. The baseline ratios that we observed, and the increase in FRET seen following removal of milrinone, are very similar to those reported previously in follicleenclosed oocytes, in which cAMP levels corresponded to $\sim 700 \mathrm{nM}$ prior to adding $\mathrm{LH}$ and dropped to $\sim 140 \mathrm{nM}$ following LH treatment (Norris et al. 2009). Oocytes treated with dynasore displayed a gradual decrease in FRET that was statistically significant after $2 \mathrm{~h}$ of treatment (Fig. 6). The YFP/CFP ratio continued to decrease even after oocytes were washed out of milrinone but maintained in dynasore. These results show that inhibiting endocytosis increases cAMP levels and suggest that signaling at the plasma membrane is needed to maintain meiotic arrest prior to the LH surge.

\section{Discussion}

Endocytosis is a ubiquitous process in somatic cells and is critically important for yolk production in the oocytes of non-mammalian species. However, endocytosis in
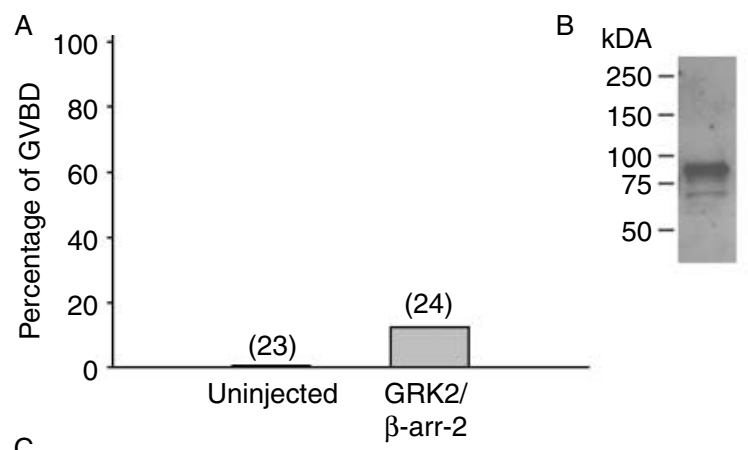

C
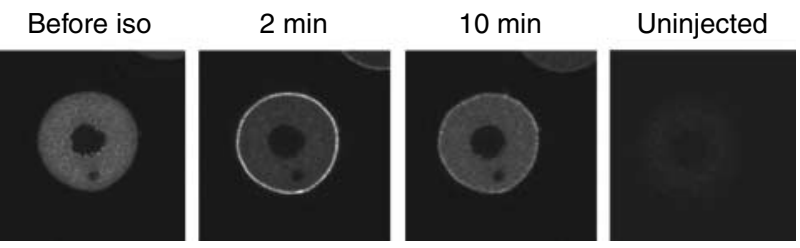

Figure 5 Effect of GRK2 and $\beta$-arrestin 2 on meiotic resumption. (A) Follicle-enclosed oocytes were injected with $G r k 2 / \beta$-arrestin-2-GFP mRNA. Follicles were cultured for 17-24 h, and the oocytes were isolated to examine meiotic status. The number above each bar is the total number of follicles per group. The experiment was repeated three times. (B) Immunoblot showing expression of GRK2 in follicleenclosed oocytes. (C) GRK2 and $\beta$-arrestin-2-GFP are active in mouse oocytes. Isolated oocytes were co-injected with GRK2, $\beta$-arrestin-2GFP, and the $\beta_{2}$-adrenergic receptor. After an overnight culture, oocytes were imaged with a confocal microscope before and after adding $10 \mu \mathrm{M}$ isoproterenol (iso). Bar $=10 \mu \mathrm{m}$. 


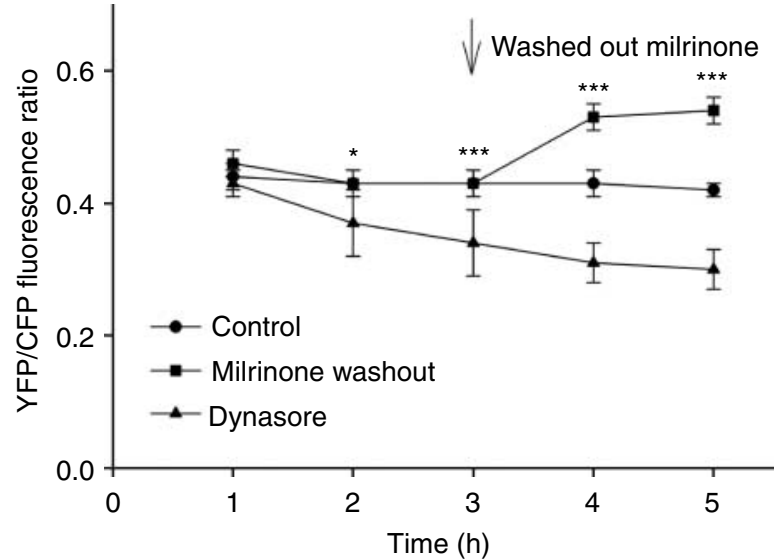

Figure 6 Inhibiting endocytosis with dynasore increases cAMP levels in oocytes. Measurement of cAMP levels in oocytes using the FRET-based cAMP sensor, Epac2-camps300. Isolated oocytes were injected with $5 \mu \mathrm{M}$ Epac2-camps300 protein, cells were visualized with a confocal microscope at hourly intervals, and the YFP/CFP ratio was measured. The $1 \mathrm{~h}$ time point corresponds to a $1 \mathrm{~h}$ treatment with dynasore. Control oocytes were maintained in milrinone throughout the course of the recordings. The arrow shows the point at which milrinone was washed out of the culture medium in the milrinone washout and dynasore-treated groups. Each point represents mean \pm s.D. Statistical significance relative to controls is indicated by asterisks $\left({ }^{*} P<0.05\right.$; $* * * P<0.0001)$.

mammalian germ cells and its possible contribution to their unique physiology is poorly understood. The aim of this study was to examine whether endocytosis occurs in the mouse oocyte and whether this affects the signaling properties of GPR3. We found that active, robust endocytosis occurs in the isolated mouse oocyte. Inhibiting endocytosis had an effect on the oocyte's meiotic status, inhibiting spontaneous meiotic resumption in the absence of PDE inhibitors. We also found that GPR3 localizes in the oocyte plasma membrane as well as in early endosomes, indicating that it is actively endocytosed in the oocyte. Inhibiting endocytosis stimulated an increase in oocyte cAMP, suggesting that signaling at the plasma membrane, possibly through an accumulation of GPR3, is responsible for maintaining meiotic arrest.

Mouse oocytes express several of the major proteins involved in receptor endocytosis, including clathrin, dynamin 2, AP-2, amphiphysin, $\beta$-arrestin- 2 , and GRK2 and GRK5. The well-characterized inhibitors of receptormediated endocytosis, MDC and dynasore, effectively prevented endocytosis in oocytes. These inhibitors blocked the formation of both endocytic vesicles and early endosomes, as well as spontaneous meiotic resumption. The effect of MDC is likely to be related to inhibition of the protein cross-linker, tissue transglutaminase (Davies et al. 1980), which is thought to inhibit the clustering and internalization of clathrin (Schlegel et al. 1982). Indeed, MDC does not inhibit endocytosis in cells lacking transglutaminase (Davies et al. 1984).
A previous study in mouse oocytes showed the presence of active tissue transglutaminase that was inhibited by MDC (Kim et al. 2001), and in that study, MDC inhibited GVBD in a dose-dependent manner. However, endocytosis itself was not examined. As a second method of blocking endocytosis in oocytes, we used dynasore. In contrast to MDC, dynasore specifically inhibits dynamin by inhibiting its GTPase activity without affecting the activity of other small GTPases (Macia et al. 2006). Dynasore may therefore be the more specific inhibitor of endocytosis. The results showing that dynasore inhibits endocytosis in oocytes support the data using MDC, confirming that endocytosis was prevented using both inhibitors.

Dynasore caused an increase in cytoplasmic cAMP levels in oocytes, suggesting that GPR3 signaling at the plasma membrane could be responsible for this increase. Recently, it has been shown that some GPRs are able to signal within endosomes (Calebiro et al. 2009, 2010, Ferrandon et al. 2009, Mullershausen et al. 2009) and that this signaling is necessary for prolonged cAMP signaling initiated by various hormones. Therefore, it is possible that GPR3 likewise signals within endosomes, perhaps to provide cAMP to the interior of the oocyte, which has a relatively large volume. Although our results do not exclude the possibility that endosomal signaling occurs within arrested oocytes, they support the hypothesis that signaling at the plasma membrane and not within endosomes is sufficient and necessary to maintain meiotic arrest.

If signaling at the plasma membrane alone is needed to maintain meiotic arrest, how is cAMP able to transmit its signal to the interior of the oocyte, which has a large volume? Cyclic AMP is a freely diffusible molecule (Bacskai et al. 1993, Chen et al. 1999), but its diffusion is restricted in many cases by the localized pools of PDEs and protein kinase $\mathrm{A}$ (PKA) tethered to the plasma membrane and various intracellular membranes by the A kinase anchoring proteins (AKAPs; Dodge et al. 2001). It is thought that PDE activity stimulated by PKA is necessary for establishing CAMP microdomains (Fischmeister et al. 2006, Houslay et al. 2007). For example, stimulation of the $\beta$-AR in neonatal cardiac myocytes generates microdomains of high concentrations of CAMP that act within a range as small as $\sim 1 \mu \mathrm{m}$, while cAMP diffuses freely in cells that have been treated with PDE inhibitors (Zaccolo \& Pozzan 2002). On the other hand, $\beta_{1}$-ARs generate cAMP signals capable of diffusing over $10 \mu \mathrm{m}$ throughout large adult ventricular cardiomyocytes even in the absence of PDE inhibitors (Nikolaev et al. 2006). AKAPs have been identified in mouse oocytes (Brown et al. 2002, 2003, Kovo et al. 2006, Newhall et al. 2006) and the RI- and RII-type PKAs are tethered in the plasma membrane and the cytoplasm respectively (Brown et al. 2002, Newhall et al. 2006, Webb et al. 2008). However, PDE activity is kept low in oocytes by cGMP, which is produced in the 
somatic cells and diffuses into the oocyte through gap junctions (Norris et al. 2009). It is not until the surge of LH that cGMP levels in the oocyte fall, allowing the activation of PDE3A (Norris et al. 2009, Vaccari et al. 2009). Therefore, it is possible that cAMP is able to readily diffuse throughout the oocyte cytoplasm prior to LH stimulation to activate intracellular PKAs. In support of this, the catalytic subunit of PKA is present both in the plasma membrane and throughout the cytoplasm (Brown et al. 2002, Webb et al. 2008).

If signaling at the plasma membrane is needed for maintenance of meiotic arrest, it is not clear why GPR3 might be targeted for endocytosis, or the mechanism(s) by which endocytosis occurs. Many GPRs undergo a conformational change upon stimulation that allows the phosphorylation by GRKs and subsequent binding and internalization by $\beta$-arrestins. In this study, we found that overexpression of $\beta$-arrestin-2 with GRK2 caused GVBD in a small percentage of oocytes within our culture period, suggesting that GRKs and $\beta$-arrestins could interact with GPR3. However, because the percentage of oocytes that underwent GVBD was not significantly different from controls, it is not likely to be the primary mechanism by which endocytosis occurs. Other GPRs (e.g. the PAR1 and $\mathrm{M}_{2}$ muscarinic receptors (Pals-Rylaarsdam et al. 1997, Paing et al. 2002) have been shown to be endocytosed through $\beta$-arrestin/GRKindependent mechanisms; it is possible that GPR3 endocytosis occurs through similar mechanisms.

Another constitutively active receptor, the herpes virus chemokine receptor US28, signals in the absence of an agonist (Casarosa et al. 2001, Fraile-Ramos et al. 2001) and could perhaps serve as a model for the regulation of the signaling of constitutively active receptors, including GPR3. US28 is constitutively phosphorylated when overexpressed in cultured cells (Mokros et al. 2002), and this phosphorylation leads to an increase in GRK/ $\beta$-arrestin-induced receptor internalization (Miller et al. 2003). In addition, overexpressing GRKs and/or $\beta$-arrestins causes a decrease in US28 activity (Miller et al. 2003). Furthermore, mutation of serines at the C-terminus impairs phosphorylation and receptor internalization, leading to an increase in surface expression and consequently to an increase in activity (Mokros et al. 2002, Miller et al. 2003). Taken together, these results show that surface expression of a constitutively active GPR is necessary for intracellular signaling. Interestingly, the internalization of US28 is enhanced by, but not dependent on, $\beta$-arrestins, although it is dependent on a clathrin-mediated pathway (FraileRamos et al. 2003, Droese et al. 2004). It will be interesting to determine whether GPR3 activity is regulated by similar mechanisms.

In summary, our results show that vesicular trafficking actively occurs at the mouse oocyte plasma membrane and that trafficking is a regulator of meiotic arrest. Although we were unable to observe an accumulation of overexpressed GPR3 at the plasma membrane following inhibition of endocytosis, our results showing an increase in cAMP concentration following inhibition of endocytosis are consistent with this possibility. In addition, our results suggest that even if GPR3 normally signals within endosomes, that signaling at the plasma membrane is sufficient for the maintenance of meiotic arrest prior to the LH surge, as CAMP levels increased following endocytosis inhibition, when endosomes were absent. It should be noted that due to the nature of the chemical inhibitors we used, which were membrane permeable, we were limited to examining isolated, rather than follicle-enclosed, oocytes. It would be interesting to determine whether inhibiting endocytosis affects LH-induced meiotic resumption in follicleenclosed oocytes. Future experiments will be needed to examine the mechanisms by which endocytosis occurs in oocytes and how GPR3 might be regulated in order to maintain the proper concentration of CAMP that is necessary to maintain meiotic arrest in the oocyte.

\section{Materials and Methods}

\section{Media and reagents}

Except where noted, all chemicals were obtained from Sigma Chemical Co. The medium used to collect isolated oocytes was MEM $\alpha$ (Invitrogen), supplemented with $20 \mathrm{mM}$ HEPES, $75 \mu \mathrm{g} / \mathrm{ml}$ penicillin G, $50 \mu \mathrm{g} / \mathrm{ml}$ streptomycin, $0.1 \%$ polyvinyl alcohol, and $10 \mu \mathrm{M}$ milrinone to inhibit spontaneous meiotic resumption. For extended culture, oocytes were placed in bicarbonate-buffered MEM $\alpha$ as above, in which the HEPES was replaced with $25 \mathrm{mM}$ sodium bicarbonate. The medium used to collect and culture follicle-enclosed oocytes was MEM $\alpha$ supplemented with $75 \mu \mathrm{g} / \mathrm{ml}$ penicillin G, $50 \mu \mathrm{g} / \mathrm{ml}$ streptomycin, and 5\% fetal bovine serum (\#12000-022, Invitrogen).

Mouse GPR3-RFP was provided by $Y$ Saeki (Ohio State University) in pHGCY and was linearized with Pacl prior to in vitro transcription using the Superscript III kit from Invitrogen. GRK2 was provided by R Lefkowitz (Duke University Medical Center) in pRK5 and was subcloned into the EcoRI and BamHI sites of $\mathrm{pBSSK}^{+}$vector. RNA was linearized with BamHI prior to in vitro transcription. $\beta$-Arrestin-2-GFP was obtained from $M$ Caron (Duke University Medical Center) in pS65T and was subcloned into pSP64.5 and was linearized with Sall prior to in vitro transcription. Rat $\beta_{2}$ AR was obtained from $N$ Ancellin (University of CT Health Center) in pBSSK + and was linearized with Notl prior to in vitro transcription. MDC was prepared as a $150 \mathrm{mM}$ stock in DMSO. Dynasore (Tocris Bioscience, Ellisville, MO, USA) was prepared as a $5 \mathrm{mM}$ stock in ethanol. FM 1-43 (Invitrogen) was prepared as a $2 \mathrm{mM}$ stock in water.

\section{Mouse oocyte and follicle isolation and culture}

All experiments were done with prior approval of the Animal Care and Use Committee at the University of Connecticut Health Center. 
Fully grown, GV-stage mouse oocytes were obtained from the ovaries of 6- to 12-week old CF-1 mice (Harlan SpragueDawley, Indianapolis, IN, USA) that had been primed with 10 IU eCG (Sigma or Calbiochem) 40-46 h prior to collection. Cumulus cells were removed by repeated pipetting through a small-bore pipette. Oocytes were cultured in $200 \mu \mathrm{l}$ drops of medium under light mineral oil on a warming tray set to $37^{\circ} \mathrm{C}$ (when in HEPES-buffered medium) or in a humidified atmosphere at $37{ }^{\circ} \mathrm{C}$ with $5 \% \mathrm{CO}_{2}$ and $95 \%$ air (when in bicarbonatebuffered medium). In some cases, the zonae pellucidae were removed using $10 \mu \mathrm{g} / \mathrm{ml} \alpha$-chymotrypsin (type II).

Antral follicles $(\geq 320 \mu \mathrm{m}$ in diameter) were dissected from the ovaries of 23- to 25-day old B6SJL/F1 mice (Jackson Laboratories) as described previously (Jaffe et al. 2009). Following isolation, follicles were plated on Millicell culture plate inserts (PICMORG50, Millipore, Billerica, MA, USA) and cultured in a humidified atmosphere at $37^{\circ} \mathrm{C}$ with $5 \% \mathrm{CO}_{2}$ and $95 \%$ air. After a $3 \mathrm{~h}$ culture period, follicle-enclosed oocytes were examined under an upright microscope for the presence of a GV. Only follicles containing oocytes with readily visible GVs were selected for use in these experiments.

\section{Microinjection}

Microinjection of isolated and follicle-enclosed oocytes was carried out as described previously (Jaffe et al. 2009, Kline 2009). For isolated oocytes, oocytes were placed in HEPESbuffered MEM $\alpha$ for microinjection. For overnight culture, oocytes were incubated in bicarbonate-buffered MEM $\alpha$ containing milrinone. Follicles were loaded into an injection chamber between two coverslips spaced $\sim 200 \mu \mathrm{m}$ apart. Following microinjection, follicles were plated on Millicell membranes and incubated for 17-24h, the oocytes were removed, and examined for their meiotic status. Quantitative microinjection was carried out using pipettes backfilled with mercury and concentrations of injected substances were calculated based on an oocyte volume of $200 \mathrm{pl}$.

\section{Immunoblotting and immunofluorescence}

For western blots, oocyte samples were made by washing oocytes in PBS containing $0.1 \%$ polyvinyl alcohol (PVA) to remove serum. Oocytes were transferred to microcentrifuge tubes, gently pelleted, and excess culture medium was removed. Oocytes were frozen in liquid nitrogen and stored at $-80{ }^{\circ} \mathrm{C}$ until use. Western blotting was performed as described previously (Mehlmann et al. 1998). Except for anti-EEA1, which was obtained from Cell Signaling Technology (Danvers, MA, USA), primary and secondary antibodies were obtained from Santa Cruz Biotechnology (Santa Cruz, CA, USA). Blots were developed using ECL Plus reagents (Amersham).

For immunofluorescence, oocytes were fixed for $1 \mathrm{~h}$ in $2 \%$ formaldehyde in $100 \mathrm{mM}$ HEPES, $50 \mathrm{mM}$ EGTA, $10 \mathrm{mM}$ $\mathrm{MgSO}_{4}$, and $0.2 \%$ Triton $\mathrm{X}-100$ at $37^{\circ} \mathrm{C}$. After fixation, oocytes were incubated in blocking buffer (PBS containing $0.01 \%$ Triton X-100, 0.1\% PVA, and 3\% BSA), then in primary antibody overnight, diluted 1:100 in blocking buffer, at room temperature. Oocytes were then washed in blocking buffer and were incubated in secondary antibody and finally in PBS containing $0.1 \%$ PVA. The secondary antibody was Alexa Fluor 488-conjugated anti-rabbit (Invitrogen). Oocytes were observed with a $40 \times, 1.2 \mathrm{NA}$ lens (C-Apochromat; Carl Zeiss Microlmaging, Inc., Thornwood, NY, USA) on either a Zeiss 510 or a Zeiss Pascal confocal microscope.

\section{RT-PCR}

RNA was extracted from oocytes or mouse brain using TRIzol reagent (Invitrogen), according to the manufacturer's instructions. cDNA was produced using the Superscript III Reverse Transcriptase kit (Invitrogen) using oligo-dT as the primer. A total of seven oocyte equivalents or $125 \mathrm{ng}$ brain cDNA was used for each PCR reaction. The cycling parameters were an initial denaturation of $2 \mathrm{~min}, 94^{\circ} \mathrm{C}$, followed by 35 cycles of $94{ }^{\circ} \mathrm{C}$ for $30 \mathrm{~s}, 55^{\circ} \mathrm{C}$ for $45 \mathrm{~s}, 72{ }^{\circ} \mathrm{C}$ for $45 \mathrm{~s}$, and a final extension at $72{ }^{\circ} \mathrm{C}$ for $7 \mathrm{~min}$. PCR products were electrophoresed on $1.5 \%$ agarose gels, visualized by staining the gel with SYBRGold (Invitrogen), and photographed with a digital camera (Canon Power Shot A650). The primer sets used for each experiment are shown in Table 1.

\section{FM 1-43 membrane labeling}

To examine endocytosis, zona-free oocytes were incubated in 2 $\mu \mathrm{M}$ FM 1-43 (Invitrogen). FM 1-43 was diluted in $\mathrm{Ca}^{2+} / \mathrm{Mg}^{2+}$-free Hank's buffered salt solution (HBSS; Gibco) containing $0.1 \%$ PVA and oocytes were examined with a Zeiss LSM 510 confocal microscope after $\sim 1-2 \mathrm{~h}$. Fluorescence was excited at $488 \mathrm{~nm}$ and was detected at $560 \mathrm{~nm}$. Images were collected using a $40 \times$ NA 1.2 water immersion objective. The method for quantifying plasma membrane and membrane to cytoplasmic ratios has previously been described in detail (supplemental material in Freudzon et al. (2005), using MetaMorph Software (Molecular Devices Corp., Downington, PA, USA) and Microsoft Excel.

Table 1 Primer sets used for RT-PCR.

\begin{tabular}{|c|c|}
\hline Dynamin 1 & $\begin{array}{l}\text { 5'-ATCTGAAGCTGCGTGATGTG-3' } \\
5^{\prime} \text {-GCCGATTCTTCCATCAGTGT-3' }\end{array}$ \\
\hline Dynamin 2 & $\begin{array}{l}5^{\prime} \text {-GACCTCCCAGGCATCACTAA-3' } \\
5^{\prime} \text {-ACTCTCGGATGTGGTTGGTC-3' }\end{array}$ \\
\hline Dynamin 3 & $\begin{array}{l}5^{\prime} \text {-GCTTGTGTGCTGTGCTTTGT-3' } \\
5^{\prime} \text {-ATCGCTGATGGATCACCTTC-3' }\end{array}$ \\
\hline AP-2 & $\begin{array}{l}5^{\prime} \text {-СTTCAAGCCСТCACTTCTGG-3' } \\
5^{\prime} \text {-TACTTGGACCTGTGGGAAGG-3' }\end{array}$ \\
\hline Amphiphysin & $\begin{array}{l}5^{\prime} \text {-GGATTCTCAGGCAACTCTGC-3' } \\
5^{\prime} \text {-TGCTCTTTCCССТСTTCGTA-3' }\end{array}$ \\
\hline$\beta$-arrestin- 1 & $\begin{array}{l}5^{\prime} \text {-CCCATGTGTGAAGGGCTAGT-3' } \\
5^{\prime} \text {-AATGACTTCACGGGACAAGG-3' }\end{array}$ \\
\hline$\beta$-arrestin- 2 & $\begin{array}{l}5^{\prime} \text {-CAACGTCCATGTCACCAACAA-3' } \\
5^{\prime} \text {-ACCACCAGCTTCACСТTGA-3' }\end{array}$ \\
\hline GRK2 & $\begin{array}{l}5^{\prime} \text {-ACCAGGGAACTGTACCGCAAC-3 } \\
5^{\prime} \text {-TGCGTTCCTTGATCTGTGTC-3' }\end{array}$ \\
\hline GRK3 & $\begin{array}{l}5^{\prime} \text {-AAGCTGGACAACGAAGAGGA-3' } \\
5^{\prime} \text {-GTGTGGAAGGCGTAGGTCAT-3' }\end{array}$ \\
\hline GRK5 & $\begin{array}{l}5^{\prime} \text {-GGTGCTGGAGACTGAGGAAG-3' } \\
5^{\prime} \text {-CCGTTAGGTCCGAACACATT-3' }\end{array}$ \\
\hline GRK6 & $\begin{array}{l}\text { 5'-ACTCTTCAGTCGCCAAAGGA-3' } \\
5^{\prime} \text {-GCCCTAGAAGCCTGGAGTTT-3' }\end{array}$ \\
\hline
\end{tabular}




\section{cAMP measurements}

CAMP measurements were made using a cAMP sensor (Epac2camps300) that has previously been described (Nikolaev et al. 2004, Norris et al. 2009). The sensor is FRET based and consists of YFP and CFP linked by the CAMP-binding domain of EPAC2. Epac2-camps300 protein $(5 \mu \mathrm{M}$ final concentration in the oocyte) was injected into isolated oocytes cultured in the presence of milrinone. Fluorescence was excited at $435 \mathrm{~nm}$ and was detected at 535/50 nm (YFP) and at 480/40 nm (CFP). Images were collected using a $40 \times$ NA 1.2 water immersion objective on a Zeiss Pascal confocal microscope. YFP and CFP intensities were quantified within a circular region of interest that was slightly smaller than the oocyte diameter using the Zeiss Pascal program. Data are reported as the ratio of YFP to CFP fluorescence after subtracting the background value from an uninjected oocyte and correcting for spectral bleed-through of CFP into the YFP channel (Norris et al. 2009).

\section{Statistical analysis}

Student's t-tests or one-way ANOVA followed by Tukey's multiple comparison post-test were performed to determine statistical significance; $P<0.05$ was considered to be significant.

\section{Declaration of interest}

The authors declare that there is no conflict of interest that could be perceived as prejudicing the impartiality of the research reported.

\section{Funding}

This work was supported by the National Institutes of Health (R01 HD056366 to L M Mehlmann).

\section{Acknowledgements}

We thank Yoshi Saeki for providing GPR3-RFP, Robert Lefkowitz for providing the GRK2 construct, Marc Caron for providing the $\beta$-arrestin-2-GFP construct, Nick Ancellin for providing the $\beta_{2}$ adrenergic receptor construct, and Laurinda Jaffe and Bruce White for helpful discussions and comments on the manuscript.

\section{References}

Bacskai BJ, Hochner B, Mahaut-Smith M, Adams SR, Kaang BK, Kandel ER \& Tsien RY 1993 Spatially resolved dynamics of cAMP and protein kinase A subunits in aplysia sensory neurons. Science 260 222-226. (doi:10.1126/science.7682336)

Barrias ES, Reignault LC, De Souza W \& Carvalho TM 2010 Dynasore, a dynamin inhibitor, inhibits trypanosoma cruzi entry into peritoneal macrophages. PLOS ONE 5 e7764. (doi:10.1371/journal.pone.0007764)

de Beco S, Gueudry C, Amblard F \& Coscoy S 2009 Endocytosis is required for E-cadherin redistribution at mature adherens junctions. PNAS $\mathbf{1 0 6}$ 7010-7015. (doi:10.1073/pnas.0811253106)

Brown RL, Ord T, Moss SB \& Williams CJ 2002 A-kinase anchor proteins as potential regulators of protein kinase A function in oocytes. Biology of Reproduction 67 981-987. (doi:10.1095/biolreprod.101.003046)
Brown RL, August SL, Williams CJ \& Moss SB 2003 AKAP7 $\gamma$ is a nuclear RI-binding AKAP. Biochemical and Biophysical Research Communications 306 394-401. (doi:10.1016/S0006-291X(03)00982-3)

Calebiro D, Nikolaev VO, Gagliani MC, de Filippis T, Dees C, Tacchetti C, Persani L \& Lohse MJ 2009 Persistent cAMP-signals triggered by internalized G-protein-coupled receptors. PLoS Biology 7 e1000172. (doi:10.1371/journal.pbio.1000172)

Calebiro D, Nikolaev VO, Persani L \& Lohse MJ 2010 Signaling by internalized G-protein-coupled receptors. Trends in Pharmacological Sciences 31 221-228. (doi:10.1016/j.tips.2010.02.002)

Casarosa P, Bakker RA, Verzijl D, Navis M, Timmerman H, Leurs R \& Smit MJ 2001 Constitutive signaling of the human cytomegalovirusencoded chemokine receptor US28. Journal of Biological Chemistry 276 1133-1137. (doi:10.1074/jbc.M008965200)

Chen C, Nakamura T \& Koutalos Y 1999 Cyclic AMP diffusion coefficient in frog olfactory cilia. Biophysical Journal 76 2861-2867. (doi:10.1016/ S0006-3495(99)77440-0)

Cheng H, Govindan JA \& Greenstein D 2008 Regulated trafficking of the MSP/Eph receptor during oocyte meiotic maturation in $C$. elegans. Current Biology 18 705-714. (doi:10.1016/j.cub.2008.04.043)

Conti M, Andersen CB, Richard F, Mehats C, Chun SY, Horner K, Jin C \& Tsafriri A 2002 Role of cyclic nucleotide signaling in oocyte maturation. Molecular and Cellular Endocrinology 187 153-159. (doi:10.1016/ S0303-7207(01)00686-4)

Davies PJ, Davies DR, Levitzki A, Maxfield FR, Milhaud P, Willingham MC \& Pastan IH 1980 Transglutaminase is essential in receptor-mediated endocytosis of $\alpha_{2}$-macroglobulin and polypeptide hormones. Nature $\mathbf{2 8 3}$ 162-167. (doi:10.1038/283162a0)

Davies PJ, Cornwell MM, Johnson JD, Reggianni A, Myers M \& Murtaugh MP 1984 Studies on the effects of dansylcadaverine and related compounds on receptor-mediated endocytosis in cultured cells. Diabetes Care 7 (Supplement 1) 35-41.

Diluigi A, Weitzman VN, Pace MC, Siano LJ, Maier D \& Mehlmann LM 2008 Meiotic arrest in human oocytes is maintained by a Gs signaling pathway. Biology of Reproduction 78 667-672. (doi:10.1095/biolreprod. 107.066019)

Dodge KL, Khouangsathiene S, Kapiloff MS, Mouton R, Hill EV, Houslay MD, Langeberg LK \& Scott JD 2001 mAKAP assembles a protein kinase A/PDE4 phosphodiesterase cAMP signaling module. EMBO Journal 20 1921-1930. (doi:10.1093/emboj/20.8.1921)

Doherty GJ \& McMahon HT 2009 Mechanisms of endocytosis. Annual Review of Biochemistry 78 857-902. (doi:10.1146/annurev.biochem.78. 081307.110540)

Droese J, Mokros T, Hermosilla R, Schulein R, Lipp M, Hopken UE \& Rehm A 2004 HCMV-encoded chemokine receptor US28 employs multiple routes for internalization. Biochemical and Biophysical Research Communications 322 42-49. (doi:10.1016/j.bbrc.2004.07.076)

El-Jouni W, Haun S, Hodeify R, Hosein Walker A \& Machaca K 2007 Vesicular traffic at the cell membrane regulates oocyte meiotic arrest. Development 134 3307-3315. (doi:10.1242/dev.005454)

Ferrandon S, Feinstein TN, Castro M, Wang B, Bouley R, Potts JT, Gardella TJ \& Vilardaga JP 2009 Sustained cyclic AMP production by parathyroid hormone receptor endocytosis. Nature Chemical Biology 5 734-742. (doi:10.1038/nchembio.206)

Fischmeister R, Castro LR, Abi-Gerges A, Rochais F, Jurevicius J, Leroy J \& Vandecasteele G 2006 Compartmentation of cyclic nucleotide signaling in the heart: the role of cyclic nucleotide phosphodiesterases. Circulation Research 99 816-828. (doi:10.1161/01.RES.0000246118.98832.04)

Fraile-Ramos A, Kledal TN, Pelchen-Matthews A, Bowers K, Schwartz TW \& Marsh M 2001 The human cytomegalovirus US28 protein is located in endocytic vesicles and undergoes constitutive endocytosis and recycling. Molecular Biology of the Cell 12 1737-1749.

Fraile-Ramos A, Kohout TA, Waldhoer M \& Marsh M 2003 Endocytosis of the viral chemokine receptor US28 does not require beta-arrestins but is dependent on the clathrin-mediated pathway. Traffic 4 243-253. (doi:10.1034/j.1600-0854.2003.00079.x)

Freudzon L, Norris RP, Hand AR, Tanaka S, Saeki Y, Jones TL, Rasenick MM, Berlot CH, Mehlmann LM \& Jaffe LA 2005 Regulation of meiotic prophase arrest in mouse oocytes by GPR3, a constitutive activator of the Gs G protein. Journal of Cell Biology 171 255-265. (doi:10.1083/jcb. 200506194) 
Hinckley M, Vaccari S, Horner K, Chen R \& Conti M 2005 The G-proteincoupled receptors GPR3 and GPR12 are involved in CAMP signaling and maintenance of meiotic arrest in rodent oocytes. Developmental Biology 287 249-261. (doi:10.1016/j.ydbio.2005.08.019)

Horner K, Livera G, Hinckley M, Trinh K, Storm D \& Conti M 2003 Rodent oocytes express an active adenylyl cyclase required for meiotic arrest. Developmental Biology 258 385-396. (doi:10.1016/S0012-1606(03) 00134-9)

Houslay MD, Baillie GS \& Maurice DH 2007 cAMP-specific phosphodiesterase-4 enzymes in the cardiovascular system: a molecular toolbox for generating compartmentalized cAMP signaling. Circulation Research 100 950-966. (doi:10.1161/01.RES.0000261934.56938.38)

Jaffe LA, Norris RP, Freudzon M, Ratzan WJ \& Mehlmann LM 2009 Microinjection of follicle-enclosed mouse oocytes. Methods in Molecular Biology 518 157-173. (doi:10.1007/978-1-59745-202-1_12)

Jovic M, Sharma M, Rahajeng J \& Caplan S 2010 The early endosome: a busy sorting station for proteins at the crossroads. Histology and Histopathology 25 99-112.

Kim SW, Lee ZW, Lee C, Im KS \& Ha KS 2001 The role of tissue transglutaminase in the germinal vesicle breakdown of mouse oocytes. Biochemical and Biophysical Research Communications 286 229-234. (doi:10.1006/bbrc.2001.5381)

Kline D 2009 Quantitative microinjection of mouse oocytes and eggs. Methods in Molecular Biology 518 135-156. (doi:10.1007/978-159745-202-1 11)

Kostenis E 2004 A glance at G-protein-coupled receptors for lipid mediators: a growing receptor family with remarkably diverse ligands. Pharmacology \& Therapeutics 102 243-257. (doi:10.1016/j.pharmthera. 2004.04.005)

Kovo M, Kandli-Cohen M, Ben-Haim M, Galiani D, Carr DW \& Dekel N 2006 An active protein kinase A (PKA) is involved in meiotic arrest of rat growing oocytes. Reproduction 132 33-43. (doi:10.1530/rep.1.00824)

Ledent C, Demeestere I, Blum D, Petermans J, Hamalainen T, Smits G \& Vassart G 2005 Premature ovarian aging in mice deficient for Gpr3. PNAS 102 8922-8926. (doi:10.1073/pnas.0503840102)

Liu YW, Surka MC, Schroeter T, Lukiyanchuk V \& Schmid SL 2008 Isoform and splice-variant specific functions of dynamin-2 revealed by analysis of conditional knock-out cells. Molecular Biology of the Cell 19 5347-5359. (doi:10.1091/mbc.E08-08-0890)

Macia E, Ehrlich M, Massol R, Boucrot E, Brunner C \& Kirchhausen T 2006 Dynasore, a cell-permeable inhibitor of dynamin. Developmental Cell 10 839-850. (doi:10.1016/j.devcel.2006.04.002)

Mehlmann LM 2005a Stops and starts in mammalian oocytes: recent advances in understanding the regulation of meiotic arrest and oocyte maturation. Reproduction 130 791-799. (doi:10.1530/rep.1.00793)

MehImann LM 2005b Oocyte-specific expression of Gpr3 is required for the maintenance of meiotic arrest in mouse oocytes. Developmental Biology 288 397-404. (doi:10.1016/j.ydbio.2005.09.030)

Mehlmann LM, Carpenter G, Rhee SG \& Jaffe LA 1998 SH2 domainmediated activation of phospholipase Cgamma is not required to initiate $\mathrm{Ca}^{2+}$ release at fertilization of mouse eggs. Developmental Biology 203 221-232. (doi:10.1006/dbio.1998.9051)

MehImann LM, Jones TL \& Jaffe LA 2002 Meiotic arrest in the mouse follicle maintained by a Gs protein in the oocyte. Science 297 1343-1345. (doi:10.1126/science.1073978)

Mehlmann LM, Saeki Y, Tanaka S, Brennan TJ, Evsikov AV, Pendola FL, Knowles BB, Eppig JJ \& Jaffe LA 2004 The Gs-linked receptor GPR3 maintains meiotic arrest in mammalian oocytes. Science 306 1947-1950. (doi:10.1126/science.1103974)

Miller WE, Houtz DA, Nelson CD, Kolattukudy PE \& Lefkowitz RJ 2003 G-protein-coupled receptor (GPCR) kinase phosphorylation and beta-arrestin recruitment regulate the constitutive signaling activity of the human cytomegalovirus US28 GPCR. Journal of Biological Chemistry 278 21663-21671. (doi:10.1074/jbc.M303219200)

Mokros T, Rehm A, Droese J, Oppermann M, Lipp M \& Hopken UE 2002 Surface expression and endocytosis of the human cytomegalovirusencoded chemokine receptor US28 is regulated by agonist-independent phosphorylation. Journal of Biological Chemistry 277 45122-45128. (doi:10.1074/jbc.M208214200)

Moore CA, Milano SK \& Benovic JL 2007 Regulation of receptor trafficking by GRKs and arrestins. Annual Review of Physiology 69 451-482. (doi:10.1146/annurev.physiol.69.022405.154712)
Mullershausen F, Zecri F, Cetin C, Billich A, Guerini D \& Seuwen K 2009 Persistent signaling induced by FTY720-phosphate is mediated by internalized S1P1 receptors. Nature Chemical Biology 5 428-434. (doi:10.1038/nchembio.173)

Newhall KJ, Criniti AR, Cheah CS, Smith KC, Kafer KE, Burkart AD \& McKnight GS 2006 Dynamic anchoring of PKA is essential during oocyte maturation. Current Biology 16321-327. (doi:10.1016/j.cub.2005.12.031)

Newton AJ, Kirchhausen T \& Murthy VN 2006 Inhibition of dynamin completely blocks compensatory synaptic vesicle endocytosis. PNAS 103 17955-17960. (doi:10.1073/pnas.0606212103)

Nikolaev VO, Bünemann M, Hein L, Hannawacker A \& Lohse MJ 2004 Novel single chain cAMP sensors for receptor-induced signal propagation. Journal of Biological Chemistry 279 37215-37218. (doi:10.1074/jbc.C400302200)

Nikolaev VO, Bünemann M, Schmitteckert E, Lohse MJ \& Engelhardt S 2006 Cyclic AMP imaging in adult cardiac myocytes reveals far-reaching $\beta_{1}$-adrenergic but locally confined $\beta_{2}$-adrenergic receptor-mediated signaling. Circulation Research 99 1084-1091. (doi:10.1161/01.RES. 0000250046.69918.d5)

Norris RP, Ratzan WJ, Freudzon M, Mehlmann LM, Krall J, Movsesian MA, Wang H, Ke H, Nikolaev VO \& Jaffe LA 2009 Cyclic GMP from the surrounding somatic cells regulates cyclic AMP and meiosis in the mouse oocyte. Development 136 1869-1878. (doi:10.1242/dev.035238)

Opresko LK \& Wiley HS 1987 Receptor-mediated endocytosis in Xenopus oocytes. I. Characterization of the vitellogenin receptor system. Journal of Biological Chemistry 262 4109-4115.

Padmanabhan S, Myers AG \& Prasad BM 2009 Constitutively active GPR6 is located in the intracellular compartments. FEBS Letters 583 107-112. (doi:10.1016/j.febslet.2008.11.033)

Paing MM, Stutts AB, Kohout TA, Lefkowitz RJ \& Trejo J $2002 \beta$-Arrestins regulate protease-activated receptor- 1 desensitization but not internalization or down-regulation. Journal of Biological Chemistry 277 1292-1300. (doi:10.1074/jbc.M109160200)

Pals-Rylaarsdam R, Gurevich VV, Lee KB, Ptasienski JA, Benovic JL \& Hosey MM 1997 Internalization of the $m_{2}$ muscarinic acetylcholine receptor. Arrestin-independent and -dependent pathways. Journal of Biological Chemistry 272 23682-23689. (doi:10.1074/jbc.272.38. 23682)

Reiter E \& Lefkowitz RJ 2006 GRKs and $\beta$-arrestins: roles in receptor silencing, trafficking and signaling. Trends in Endocrinology and Metabolism 17 159-165. (doi:10.1016/j.tem.2006.03.008)

Schlegel R, Dickson RB, Willingham MC \& Pastan IH 1982 Amantadine and dansylcadaverine inhibit vesicular stomatitis virus uptake and receptor-mediated endocytosis of $\alpha_{2}$-macroglobulin. PNAS $\mathbf{7 9}$ 2291-2295. (doi:10.1073/pnas.79.7.2291)

Vaccari S, Horner K, Mehlmann LM \& Conti M 2008 Generation of mouse oocytes defective in CAMP synthesis and degradation: endogenous cyclic AMP is essential for meiotic arrest. Developmental Biology 316 124-134. (doi:10.1016/j.ydbio.2008.01.018)

Vaccari S, Weeks JL, Hsieh M, Menniti FS \& Conti M 2009 Cyclic GMP signaling is involved in the luteinizing hormone-dependent meiotic maturation of mouse oocytes. Biology of Reproduction 81 595-604. (doi:10.1095/biolreprod.109.077768)

Webb RJ, Tinworth L, Thomas GM, Zaccolo M \& Carroll J 2008 Developmentally acquired PKA localisation in mouse oocytes and embryos. Developmental Biology 317 36-45. (doi:10.1016/j.ydbio.2008.01.045)

Wolfe BL \& Trejo J 2007 Clathrin-dependent mechanisms of G proteincoupled receptor endocytosis. Traffic 8 462-470. (doi:10.1111/j.16000854.2007.00551.x)

Yin H, Chu A, Li W, Wang B, Shelton F, Otero F, Nguyen DG, Caldwell JS \& Chen YA 2009 Lipid G protein-coupled receptor ligand identification using $\beta$-arrestin PathHunter assay. Journal of Biological Chemistry 284 12328-12338. (doi:10.1074/jbc.M806516200)

Zaccolo M \& Pozzan T 2002 Discrete microdomains with high concentration of CAMP in stimulated rat neonatal cardiac myocytes. Science 295 1711-1715. (doi:10.1126/science.1069982)

Received 12 November 2010

First decision 5 January 2011

Revised manuscript received 11 March 2011

Accepted 16 March 2011 